\title{
A biomimetic study of natural attachment mechanisms-Arctium minus part 1
}

Bruce E. Saunders

\begin{abstract}
The hook as a shape occurs very early on the evolutionary scale. Cellulose appears as a biological building block in the Cambrian age from fossil records, and hooks have been found in chitinous organisms as mandibles, maxillae as well as tarsi. Hook separation forces associated with plant seed and fruit dispersal have been studied in four species by SN Gorb. A fifth, Arctium minus (or Burdock as it is commonly known) is purported to have been the source of engineering design inspiration for George de Mestral (see Velcro). There are marked differences in the shape and functionality of natural A. minus hooks, and the probabilistic fastener that he designed and developed, namely the needle-like profile for insertion of the A. minus hooks, their lack of reusability and their physical size. In functionality, Velcro resembles that of Circaea lutetiana, not A. minus. From the study of SN Gorb's data, it is concluded that there are additional indicators for structural behaviour as well as the morphological variables indicated by Gorb, and these are flexible versus fixed bases to the shafts and degrees of resilience (stiffness, the inverse of Young's modulus) of the component material. The natural substrate properties are presented as being indicative of the receptiveness of the hooks to a range of substrates. Field testing consisted of tensile testing mounted $A$. minus hooks in an Instron tensile tester in a laboratory to note the fracture strength (contact separation force) and mode of failure which was characteristic of a composite biomaterial. The study indicates that contrary to Gorb, the reduction in the size rendered the bending moment due to the hook span to be negligible and of little effect. Note that an early original botanical paper on A. minus is missing presumed lost from the British Library and that finite element analysis follows in paper III, and an experiment into the use of confocal microscopy (shape acquisition) in paper II. Results indicate that the contact separation force is independent of bending moment and the "span" of the hook, a scaling effect that is important for the design for manufacture (DFM) of micron range-sized hooks.
\end{abstract}

Keywords: Micron-sized; Cladistics; Tensile testing; Fracture; Bio-composite; Cellulose; Biological design indicators; Scaling effects; Design for manufacture

\section{Background}

This series of papers draws upon the work of SN Gorb and his colleagues due to the loss of the paper on Arctium minus from the British Library. A. minus (Fig. 1) is a species of plant that supports hooks for the purposes of seed dispersal that was omitted in his study of scaling effects [1] in biological hooks and it is studied here. Further, after the study of the Galium aperine hook [2], the hook of the A. minus is studied to identify any salient biological design indicators that could give rise, biomimetically, to the development of a new product or surface structure.

Correspondence: brucesaunders23@hotmail.co.uk University of Bath, 54 Ballance Street, Bath, UK
The question of intelligent design and perfection in plant hooks associated with plant reproduction is taken to have been addressed by the work of Allmon and Ross as cited by Nicklaus [3], Howe and Smallwood [4] and by SH Bullock [5], and it is therefore sufficient to merely state here that for the purposes of structural biomimetic study, plant biological structures must be treated as they are without regard to their origins or reason for being and examined for design indicators that can be utilised for the purposes of modern design and manufacture. To assume perfection in nature's design is a fallacy. All that can be assumed about nature's designs is energy efficiency with available materials. However, in seeking a design indicator, successful structures that are present in

\section{穴}




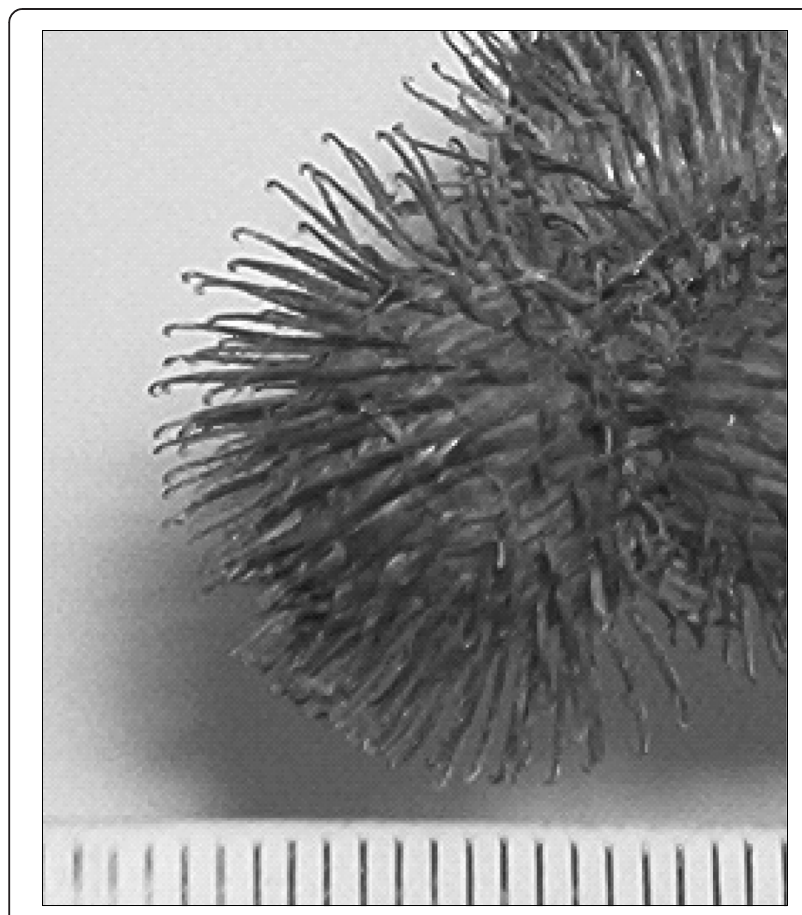

Fig. 1 Arctium minus

more than one species are a point of departure for study from Nicklaus' analysis of the evolutionary process (i.e. the structures have passed through the "evolutionary sieve" in more than one species-an indicator of success).

SN Gorb states [1] that at the time of his writing, there were no commercial lightweight attachment mechanisms that exhibited a flexible base as he found in the case of G. aperine (multiple degrees-of-freedom). This can be expanded to include probabilistic and fields-of-hooks fasteners. Species he studied that support hooks were Agrimonia eupatoria, Circaea lutetiana, G. aperine and Geum urbanum. These hooks are not formed through the process of adaptive growth since they are single-use. They therefore must be genetically defined to occur in their shape. Interaction with the environment changes their qualities in a genetically predefined manner. The distinct difference between these hooks is the structures from which they arise; G. aperine hooks are stomatal in origin as are those of A. eupatoria and C. lutetiana while the hooks of G. urbanum arise from carpels. Only the hooks of A. minus arise from modified bracts encasing the ovary.

\section{Scale}

D'Arcy Wentworth Thomson's book On Growth and Form [6] devotes a chapter to scale effects. In the case of natural-hooked structures, many of the examples in nature are small $(\sim 100 \mu \mathrm{m}$ in thickness) so that scaling factors become significant in the action of attachment. It is common to find that it is a combination of properties that act coincidentally that produce an effect [7].

Adhesive secretions, other fluid properties such as surface energy and capillarity and applied pressure gradients combine with mechanical interlock to produce a resultant attachment force. Gorb notes [8] that biological systems present the material scientist with goals for new materials that can model the behaviour of biomaterials and this work anticipates advancements in artificial cellulose.

The burdock hook (span $\sim 250 \mu \mathrm{m}$, shaft diameter $\sim 200 \mu \mathrm{m})$ is desiccated when mature, without any secretory organs. The full action of mechanical interlock can take place in both a wet and dry environments; thus, the scaling effects are limited to those of size, friction, moisture, inertia and gravity.

It can be predicted that there will be a problem using conventional rapid prototyping devices to judge their performance due to their inability to reproduce resin models in the order of size of less than $100 \mu \mathrm{m}$ in thickness which is necessary in order to reproduce any scaling effects observed in the biological sample. A typical deposition prototyper deposits nylon resin in layers of $100 \mu \mathrm{m}$, about half the burdock shaft thickness, and further, the material lacks properties analogous to biological materials [9]. Similar commercial finite element analysis (FEA) tools such as Solidworks 2004 lack facilities for composite study.

\section{The functional ecology of $A$. minus}

Higher plants use a variety of dispersal agents such as wind, water, animals and people [1]. Dispersal by animals is known as zoochory. The dispersal of seeds or fruit (known as diaspores, more often fruit than seeds) by attachment to animal fur or feathers is known as epizoochory. Diaspores of this kind do not provide valuable nutrition to the animal to which they attach themselves nor do they actively attract animals to parent plants. Instead, they have special structures such as hooks, barbs, burrs and spines or sticky secretions, and they detach easily from the parent plant.

A. minus is commonly known as burdock, and it is found throughout the UK and is a member of the thistle family. It is common knowledge that it is an annual noxious weed commonly found by the side of pathways and riverbanks. It grows approximately to $2 \mathrm{~m}$ in height and generally features single or multiple primary stems off which arise secondary and tertiary branches.

In fur and feathers, the diaspores may remain attached for a long period of time until animals groom them off or until the animal dies. A. minus has natural symbiotic partners in seed dispersal that are wild animals and birds indigenous to the UK, such as rabbits, badgers, foxes, 
sheep and deer. The diaspores of $A$. minus are adapted for dispersal by mechanical interlocking.

In terms of the plant's life cycle, the hooks become operational early in the year, acting as a defense mechanism while the immature seeds develop. From observation, at this stage, the tensile force required to remove the fruit from its supporting stem is at its highest. The corolla or flowers are in evidence at the apex of the fruit, protruding from the basal cup comprising of the ovary and surrounding bracts. This fruit is green and the hooks are developed. As the fruit matures, the corolla withers and then disappears. The seeds are present in the ovary, and these are freed by the total disintegration of the fruit which begins immediately when the fruit is separated from its host plant with the use of the now brown and dry bract hooks.

Each of the bracts is flattened at the base where it originates, becoming narrower to form the shaft of the hook. Therefore, each hook has a single degree of freedom which, Gorb [1] says, decreases the contact separation force and increases the propensity of the fruit to attach because the ability to bend implies weaker and flexible cell structures yet a greater ability to become attached in a probabilistic manner. This is not the case for A. minus. As the plant and its seeds mature, the entire plant desiccates and becomes brittle. The detachment forces required from its supporting stem for the now brown fruits and the mature seeds they contain reduce to loads far below that of the fracture forces of the hooks and the fruit freely attaches itself to passing host. This is a one-off attachment. Once the fruit makes contact with the ground, it is ready to await germination.

Due to its narrow profile, the burdock hook has a further function, namely an insertion effect. It enables the hook to pierce fibrous surfaces like a needle, and this could be an important indicator in later design work.
The behaviour of the A. minus hook under loading is studied here. The hook is composed of the biocomposite cellulose which is comprised of cellulose fibrils bound together in a matrix of hemi-cellulose and lignin, [10] and [11].

\section{The substrate}

The substrate, because it forms one half of the attachment system for these types of hooks, must be accounted for in a study for the purposes of producing a product. The question that needs to be answered is the overall effect the substrate fulfills. In terms of the host substrate being fur or feathers, the qualities of these substrates have been studied elsewhere for other purposes.

Hair is made of keratin. Keratins form a group of varied proteins which contain significant amounts of sulphur cross-linking and stabilizing in the material. It is found in horn, hair, hoof, feather, skin, claws etc. The types of keratin include mammalian, avian and others such as reptilian [10].

In mammals, keratin occurs in hair, hoof and horn. Human hair is a composite consisting of a fibre/matrix mix. In a relaxed state, the hair is mainly $\alpha$-form and when heated and pulled straight, it turns into a $\beta$-form. The $\alpha$-form is the $\alpha$-helix and the $\beta$-form is the antiparallel $\beta$-sheet which results from pulling the $\alpha$-helices beyond their yield point. Bird feathers are also made of keratins as is silk.

This can be related to the inner radius of the hook in order to seek design indicators of an optimum ratio. Such experimentation can be left until later in the product design, but for the moment, it should be mentioned that the span of the burdock hook, from outer tip to inner shaft surface, is approximately $250 \mu \mathrm{m}$, and this can be compared to the above figures (see Table 1).

Table 1 Results of tensile testing

\begin{tabular}{|c|c|c|c|c|c|c|}
\hline \multirow[t]{2}{*}{ Hook number } & \multicolumn{6}{|c|}{ Fruit specimen number } \\
\hline & 1 & 2 & 3 & 4 & 5 & 6 \\
\hline 1 & 0.00101 & 0.00114 & 0.00057 & 0.00135 & 0.00059 & 0.00139 \\
\hline 2 & 0.00104 & 0.00121 & 0.00108 & 0.00093 & 0.0011 & 0.00114 \\
\hline 3 & 0.00107 & 0.00099 & 0.0011 & 0.00112 & 0.0012 & 0.00112 \\
\hline 4 & 0.00103 & 0.00095 & 0.00083 & 0.00124 & 0.00088 & 0.00106 \\
\hline 5 & 0.00087 & 0.00098 & 0.00092 & 0.00114 & 0.00109 & 0.00105 \\
\hline 6 & & 0.00085 & 0.00091 & 0.001 & 0.00118 & 0.00131 \\
\hline 7 & & 0.0009 & 0.00092 & 0.00086 & 0.00127 & 0.00127 \\
\hline 8 & & 0.00093 & 0.00107 & 0.00113 & & 0.00109 \\
\hline 9 & & 0.0009 & 0.00115 & & & 0.0012 \\
\hline Average (N) & 0.001 & 0.000983 & 0.00095 & 0.001096 & 0.001044 & 0.001181 \\
\hline Standard deviation & $7.8 \mathrm{E}-05$ & 0.000118 & 0.000179 & 0.000161 & 0.000235 & 0.000119 \\
\hline Diameter (mm) & 23.5 & 15 & 14 & 26 & 25 & 23 \\
\hline
\end{tabular}


The most important aspect to note with reference to the functionality of $A$. minus is that there is not a critical geometrical relationship between the hook and a particular fibre. The hook accepts all fibre diameters for the purposes of mechanical interlock, i.e. it is nonspecific.

\section{Methods}

Tensile testing of the $A$. minus hook

The testing of the burdock hooks occurred in laboratory conditions. The hooks of burdock fruit were harvested a month prior to testing and stored in a dry condition. The purpose of this experiment was to study the material behaviour for the purpose of designing a new product/structure. Certain aspects of the bract's behaviour were isolated for the purpose of the experiment whilst others were suppressed. The detachment force of the fruit from the stem, the extension of the hook prior to fracture and the force to remove the hook from the fruit were all suppressed for the purposes of the experiment. The first and third because they were irrelevant to the study, and the second because the hooks were all naturally curved prior to tension being applied making it difficult to isolate true extension due to material deformation from extension due to the taking up of slack. Further, standard values of Young's modulus for cellulose (7-15 GPa from [10]) were used. This was not an experiment to determine the Young's modulus, and the measured strain was not relevant to the test.

\section{Aims}

The aim of this study is to investigate the fracture force and mode of fracture of hooks from the plant genus $A$. minus or common Burdock using an Instron tensile testing machine to observe any telling differences that could be observed from the conclusion of Gorb that the span of the hook was the significant factor in contact separation force by testing specimens of different radiuses of hook collected from burdock pods of varying diameters. Further aims are to observe the nature of fracture of the composite biomaterial and to use this information in developing a design for an attachment mechanism/structure based upon the burdock hook and its form and functionality.

This experiment draws from the methods and is compared with the results of the following two papers:

1. Natural hook-and-loop fasteners: Anatomy, Mechanical Properties and Attachment Force of the Jointed Hooks of the Galium aparine Fruit by EV Gorb, VL Popov, and SN Gorb [2], and

2. Contact Separation Force of the Fruit Burrs in Four Plant Species Adapted to Dispersal by Mechanical Interlocking by EV Gorb and S Gorb [1].
These two papers yield fundamental descriptions and conclusions which the following experiment is set out to investigate. Their investigation into scaling effects in small hooks led to the following assertions: the four main attributes that influence burr performance are span, structure, size and material flexibility. All four species tested were found to exhibit behaviour within the known bounds of cellulose performance. ( $E=7-$ $15 \mathrm{GPa}$ ). The significant difference detected was an unforeseen weakness in strength displayed by C. lutetiana. This was associated with an increase in material flexibility; the hooks did not fracture, they flexed to release the loop and the hooks remained intact. This form of response is very similar to that of commercial Velcro and would indicate that Velcro better approximates the behaviour of $C$. lutetiana than A. minus as is commonly asserted.

Gorb suggests that it would be of interest to investigate the required force for detachment of the fruit from the stems, but this was not considered relevant to this method for designing a biomimetic fastener.

\section{Method and apparatus}

1. Specimens were collected from four separate burdock plants that grow behind the University of Bath accommodation blocks. The plants all stand in a line next to a sandy path that passes between the university grounds and the golf course.

2. Note was maintained of the conditions of collection and the regions of the individual plants from which specimens were collected. These specimens were collected late in October 2003 and tested in December. It was observed that the plants themselves were brown and dry with the leafy vegetation of early season growth disappeared and the seedpod fully developed.

3. It was judged that the effect of the delay between the collection and testing of the hooks would have little effect on the relative performance of the hooks and probably little effect on the absolute performance of the individual hooks given that they were collected in a naturally desiccated state and maintained in a dry condition until ready for testing, thereby preventing/inhibiting decomposition. Their desiccated state also made them ready for SEM work.

4. Five individual fruit specimens (each consisting of an array of approximately 100 hooked bracts) were collected from each plant giving 20 specimens in total. A selection of these specimens was then tested.

5. The Instron tensile tester was equipped with a 1-N load cell. 


\section{Specimen preparation}

Each fruit was sectioned into halves under a dissecting microscope. One of these halves was returned to the specimen packet in case more hooks from the same specimen would be required. The other hemisphere of bracts/ovary/seeds was separated to yield individual hooked bracts for experimentation.

Ten individual hooks were taken from the dispersed hemisphere. These were mounted in preparation for testing in the Instron machine by gluing each separate bract to a plastic mounting (Fig. 2). The bract shaft with its hook extended was exposed for interaction with a testing substrate analogue, a loop of silk thread. The Instron tensile tester applies extension and measures the resultant reaction in the specimen through the load cell, and the rate of extension was set at $1 \mathrm{~mm} / \mathrm{s}$ using a $1-\mathrm{N}$ load cell (Fig. 3).

Gorb states that the shaft length is the principal main morphological variable influencing a hook's strength. Thereafter, hook span and material flexibility are most important, his theory being that the larger the span, the greater the lever arm of the bending moment and the stress at the shaft. In this case, material composition is constant for all the hooks.

\section{Results}

The results of the tensile testing are tabulated below (Table 1). Up to nine sample hooks were taken off of six fruit. The mean value of these results for each specimen is plotted versus the diameter of the fruit (Fig. 4).

The results of this tensile test were directly compared with the values obtained by Gorb in his paper on contact separation forces. In his experiments, the species with the lowest contact separation force is C. lutetiana, the species whose hook functionality most closely matches the flexible hooks of Velcro which are flexible in order to meet the demand of multiple usage in the product with the minimal of damage to hook and fibre substrate

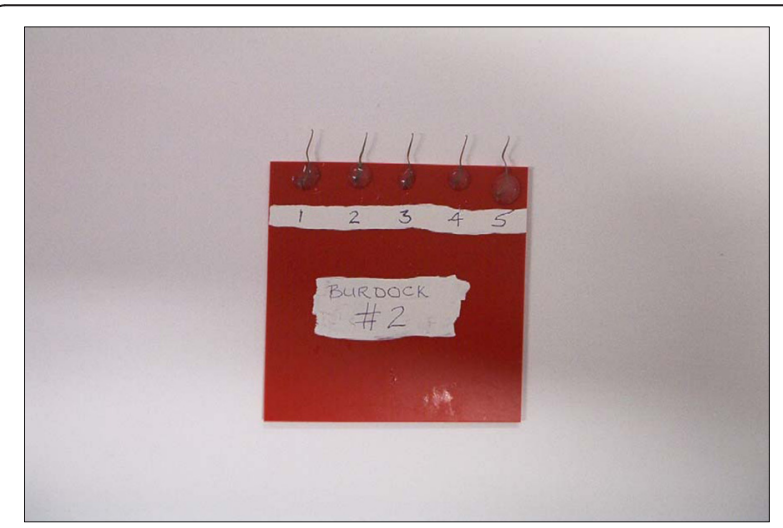

Fig. 2 Hooks mounted in preparation for testing in the Instron machine by gluing each separate bract to a plastic mounting

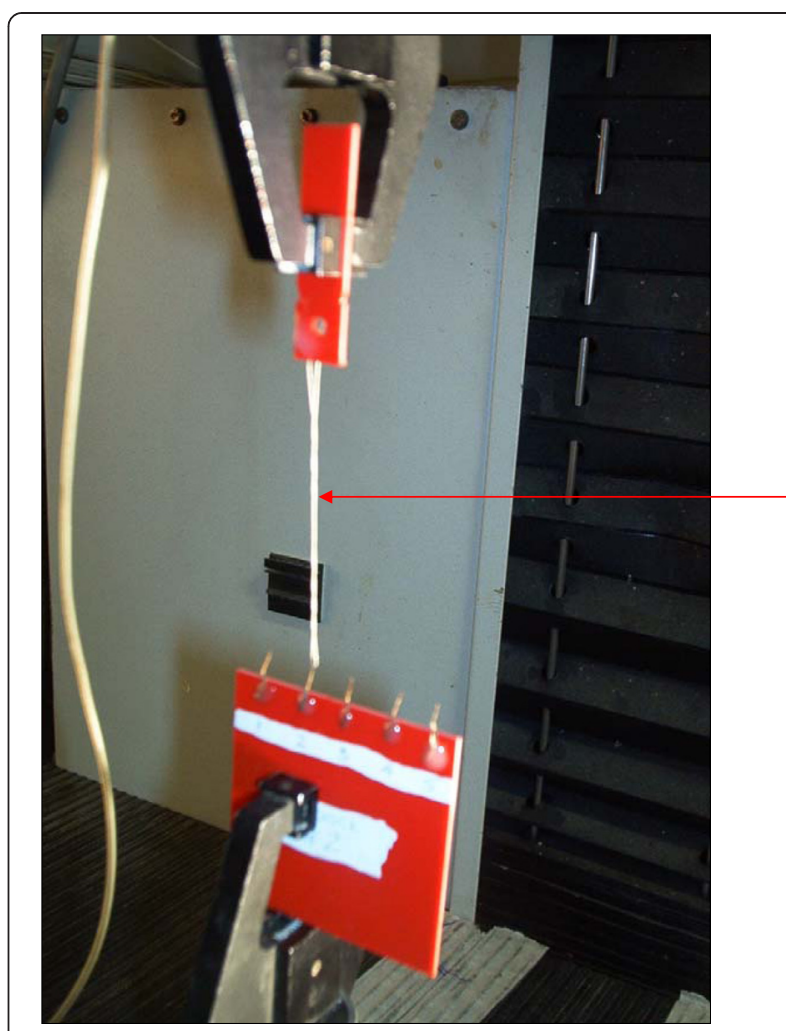

Fig. 3 The Instron tensile tester applies extension and measures the resultant reaction in the specimen

(for clarity, note that there is a difference between flexible bases and flexible hooks-in the first instance, the whole structure moves with the flexing of the base, in the second, only the arch of the hook flexes).

Comparing the force results obtained for A. minus with Gorb's results shows that the magnitude of the results for A. minus fall between those of $A$. eupatoria and G. urbanum but are significantly higher than the other species with a flexible base, G. aperine. Certainly in terms of order of magnitude, the tests would seem to reflect a fair result.

Therefore, the order of magnitude in fracture strength, from lowest to highest with $A$. minus inserted in place and labelling each species according to its structural composition, is as follows (Table 2), that is, in increasing cellular complexity and thickness. Gorb's investigation into his four hooked species was to investigate the correlations between contact separation force and various morphological variables. The G. aperine hook was of interest to Gorb since these hooks were distinguished by their hollow base and high degree of flexibility in all directions. Flexibility is also found in $A$. minus with the flattened bract at the base of the hook.

In terms of statistical analysis, Gorb used ANOVA based upon ranks to compare the variables of the four species and investigate the correlations. In the case of 


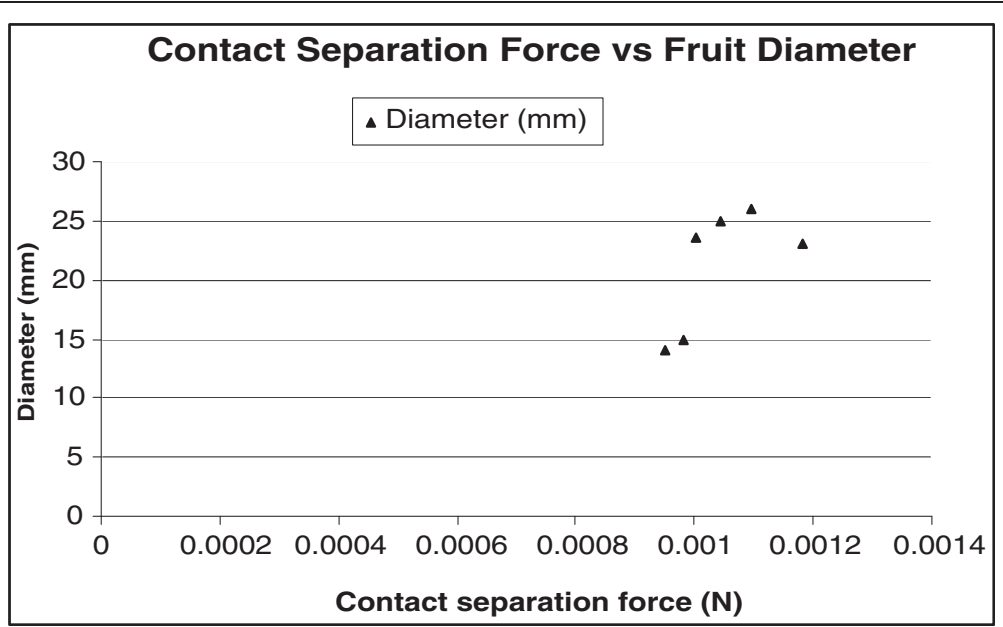

Fig. 4 Mean value of these results for each specimen is plotted versus the diameter of the fruit

this experiment where a single species is investigated, it is suited to use a direct comparison between the load values of his paper. A simple form of scale measurement is used, that those hooks that originate from a large fruit would be proportionately larger than those from a small fruit. This is, in fact, obviously true from visual inspection of the specimens.

\section{Images of fracture}

The SEM images of the fractured hooks in Fig. 5 clearly show the fibrous nature of the hook material. The fracture surfaces are of interest. As will be seen in Part II, the hooks all have a thickening of the outer surface in the early curvature of the hook which will resist bending and compression, inducing fracture instead of bending. This effect is visible in the images.

The composite nature of the material is clear, and it should be noted how the fracture surface fractures unevenly and there is fibre "pull-out". From [10], fibre "pull-out" indicates that the fibre/matrix interface of the plant material consisting of cellulose microfibrils, hemicellulose and lignin is tightly bound. It can be seen from Fig. 5 that all four specimens (taken to be typical examples of fracture in the specimen set) experienced failure on the inner curvature of the hook as would be expected for a hook of material experiencing a bending moment with the inner fibres under tension while the external fibres are in a state of relative compression about a point

Table 2 Hook Structure type

\begin{tabular}{lcc}
\hline Stomatal & Bract & Carpel \\
\hline C. lutetiana & A. minus & G. urbanum \\
G. aperine & & \\
A. eupatoria & & \\
\hline
\end{tabular}

of rotation. More importantly for a hook shape, the inner fibres will be shorter than the outermost fibres therefore strain will be highest in the inner fibres. This means that the tensile forces in the inner curvature and therefore induced shear will be higher than the fibres in the outer curvature.

The surface is typical of a fibrous composite break in bending. All hooks fractured at the region of the join between hook and shaft which is the region of the entire hook structure (both shaft and arced tip) that experiences the largest stress when the hook is placed in tension, noting that the bract itself is secured by glue. At no single instance did any of the test hooks fail at the bract.

\section{Analysis}

It was noted that there was a difference between the diameter of wire used by Gorb and the silk thread of this experiment (of the order of $\times 10$ ). Both the wire loop and the silk thread represent an artificial substrate, replacing natural fibre. Silk thread is a composite of natural silk of which each fibre is much finer than fur fibres. The wire loop appears much finer than a natural fibre, and the question should be asked if it contributed to severing the hook tips.

The analysis that follows derives from undergraduate engineering structural analysis. Note that although cellulose is a biocomposite, the density of the fibres in the fibre/matrix composite is such that for the purposes of analysis the hook material shall be treated as an isotropic solid. This is because the hook fibres are loaded in direct stress only. The basic morphology of the hook shall follow in the diagram of Fig. 7. Dimensions for the purposes of the 2-D analysis, for the hook, are taken from the SEM (Fig. 6). FEA follows in Paper III. 

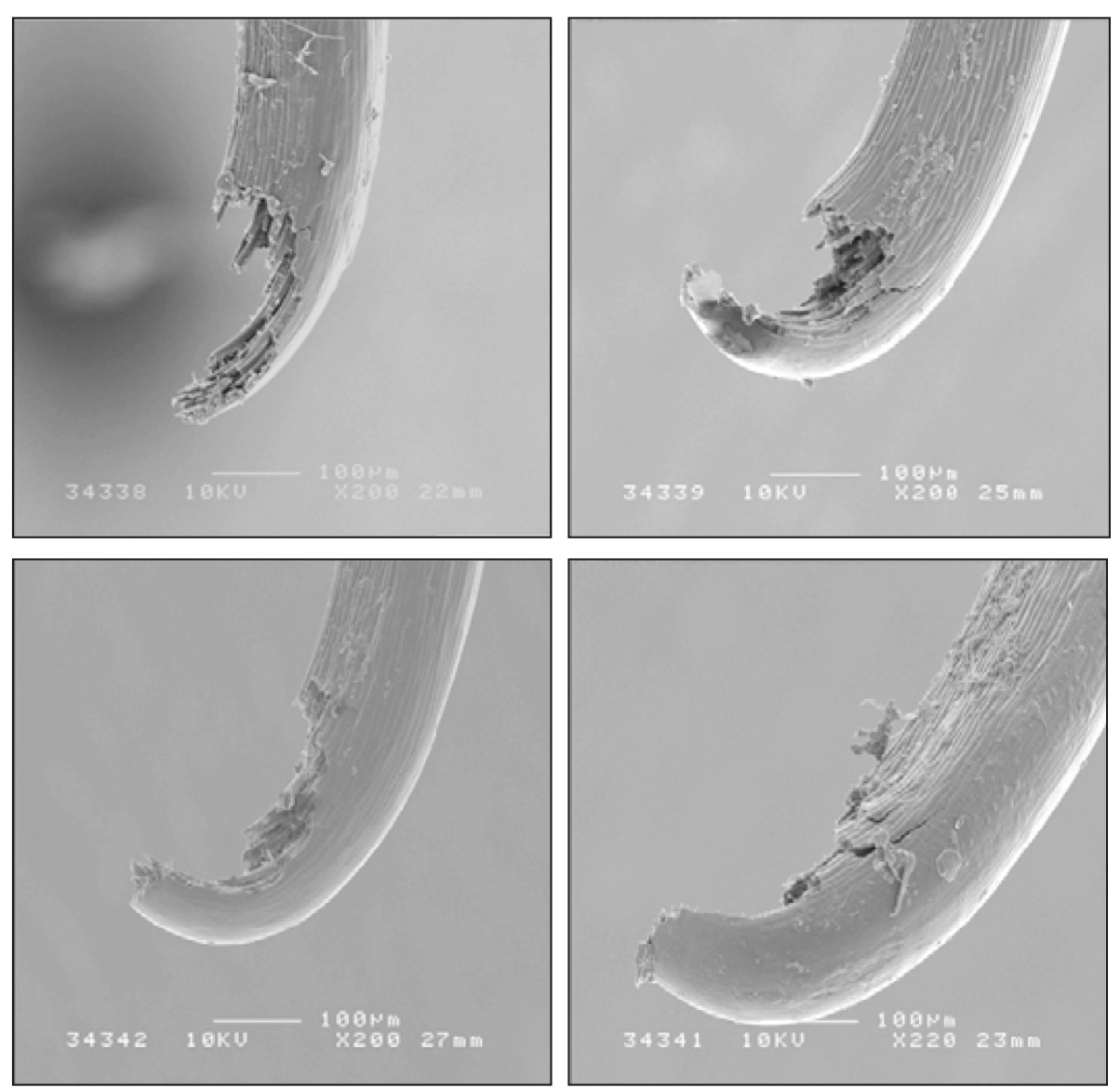

Fig. 5 SEM images of the fractured hooks

Let the hook be placed under tension. The stresses in the material will comprise two components due to the tensile loading, a tensile stress and a shear stress on the line of fracture due to the tensile loading (Fig. 7).

It has been noted previously that visually the $A$. minus hook does not taper in diameter from the top of the shaft. There is an increase in material on the shoulder of the hook before it tapers to a point. This influences the hook's behaviour in tension, increasing its resistance to flexing, increasing its attachment to its host and its disposition to fracture, not bend.

The analysis of a 2-D hook under loading is completely presented in Fenner [12].

Vincent has presented standard figures for the elastic modulus of cellulose as 7-15 Gpa and notes that for biomaterials the Poisson ratio is generally taken to be 0.5 (though not always).

By using these figures as maximum and minimum values and calculating the stress due the loading, it should be possible to evaluate the percentage strain during the test. All dimensions are taken from Fig. 6. Calculations are based upon the sketches in Fig. 7. The question that Gorb does not answer is whether the hook fails under direct stress or through shear. This is evaluated below. The values for direct strain and shear strain are calculated and compared. This specimen calculation is based upon the average fracture force of specimen 3. The following are the experimental values:

$f=0.001168 \mathrm{~N}$ (from Table 1)

Span $=250 \mu \mathrm{m}$

Diameter of shaft $=D=200 \mu \mathrm{m}$

$$
E=\text { elastic modulus }=s / e=\text { stress } / \text { strain }
$$

$=[7,12]$ Gpa from $[10]$

$$
G=\tau / \gamma=E /[2(v+1)]
$$

$v=0.5$ (an approximation from [10]) 

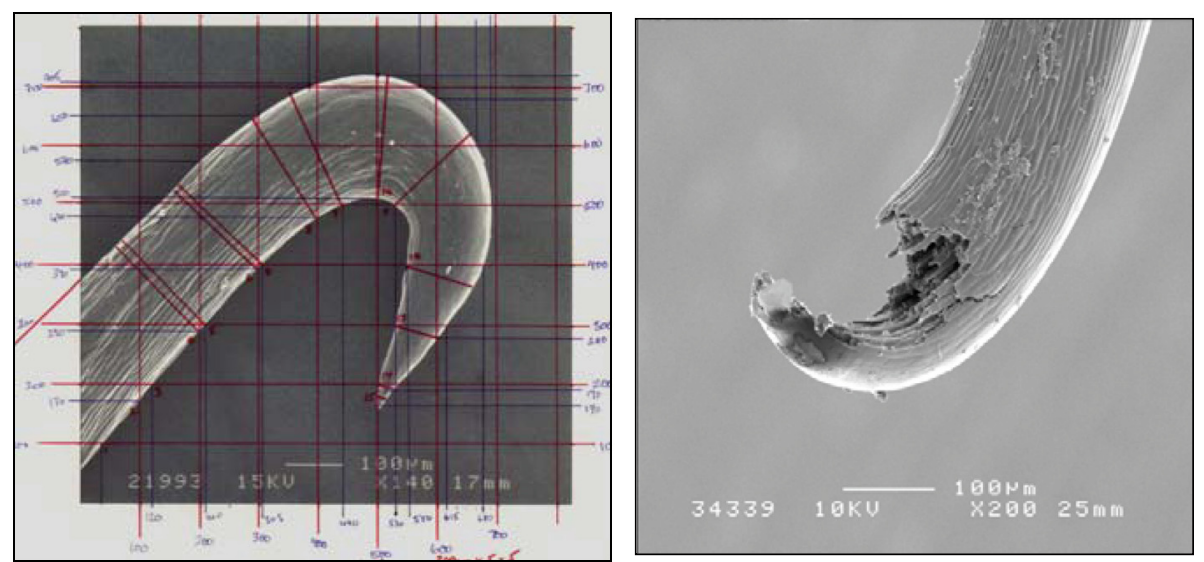

Fig. 6 Dimensions for the hook

$$
\begin{aligned}
B= & L=\text { Lever Arm } \\
= & \operatorname{Span} / 2+D / 2 \\
& +y \quad \text { From the beam geometry } \\
\sigma_{x}= & F / A-y M / I \quad \text { From the beam geometry }
\end{aligned}
$$

$I=\pi D^{4} / 64 \quad$ Second moment of area of a circular beam

$\sigma_{t}=$ direct tensile stress $=$ force $/$ area $=f / A$

where the area is the cross-section of the hook perpendicular to the longitudinal axis.

$$
A=(\pi * d * * 2) / 4
$$

where $d=200 \times 10^{-6}$

$$
\mathrm{L}=\text { lever } \operatorname{arm}=\mathrm{d} / 2+\operatorname{span} / 2
$$

Span $=250 \mu \mathrm{m}$, therefore

$$
L=225 \mu \mathrm{m}
$$

$$
\sigma_{t}=f / A=f * 4 /\left(\pi * d^{2}\right)
$$

from (4)

$$
\begin{aligned}
\sigma_{t} & =\left(1.17 \times 10^{-3} \times 4\right) /\left(\pi \times\left(200 \times 10^{-6}\right)^{2}\right) \\
& =37.24 \mathrm{MPa} \\
\sigma_{\mathrm{bm}} & =f * L * y_{\mathrm{NA}} / I_{\mathrm{yy}}
\end{aligned}
$$

from (4)

where $I_{\mathrm{yy}}=$ the second moment of area of a circle about a neutral axis $y-y$ and $y_{\mathrm{NA}}$ is the distance from the neutral axis to the edge of the section.

$$
\begin{aligned}
\sigma_{\mathrm{bm}} & =\left(100 \times 10^{-6} \times 225 \times 10^{-6} \times 1.17 \times 10^{-3} \times 4\right) \\
& /\left(\pi \times\left(200 \times 10^{-6}\right)^{2}\right) \\
& =0.84 \times 10^{-3} \mathrm{~Pa}
\end{aligned}
$$

from (4)

Bending stress at failure is negligible compared to direct stress.

$$
\sigma_{\text {total }}=37.24 \mathrm{MPa}
$$

from (4)

Now by substituting each of the range values of $E=$ (7$15 \mathrm{GPa}$ ) for cellulose we find $\varepsilon_{\min }$ and $\varepsilon_{\max }$.

$$
\varepsilon_{\max }=0.00532, \quad \varepsilon_{\min }=0.00248
$$

from (2)

These results suggest that the bending moment has a negligible impact upon the failure in direct stress. Therefore, there is a scaling effect such that the span of the hook becomes of little consequence relative to the material properties of the hook. This has a direct impact upon DFM.

If we consider shear stress for a homogenous material, $\sigma_{\mathrm{s}}=F_{\mathrm{s}} / \underline{A}$

where $\bar{A}$ is the angled shear plane at angle $\theta$ and $\mathrm{F}_{\mathrm{s}}$ is the shear component of the applied load, then

$$
\begin{aligned}
& F \cos \theta / A \sec \theta=\tau_{\mathrm{s}} \\
& \begin{aligned}
\left.\theta=0^{\circ} \text { (by inspection from SEM }\right) \\
\text { Then, } \begin{aligned}
\tau_{\mathrm{s}} & \left.=\left(\left(1.17 \times 10^{-3}\right) \cos \theta\right) /((\pi \times d * * 2) \times \sec \theta / 4)\right) \\
& =37.24 \mathrm{MPa}
\end{aligned} \\
\text { If } G=E /(2 \times(1+v))
\end{aligned}
\end{aligned}
$$

from (2) 


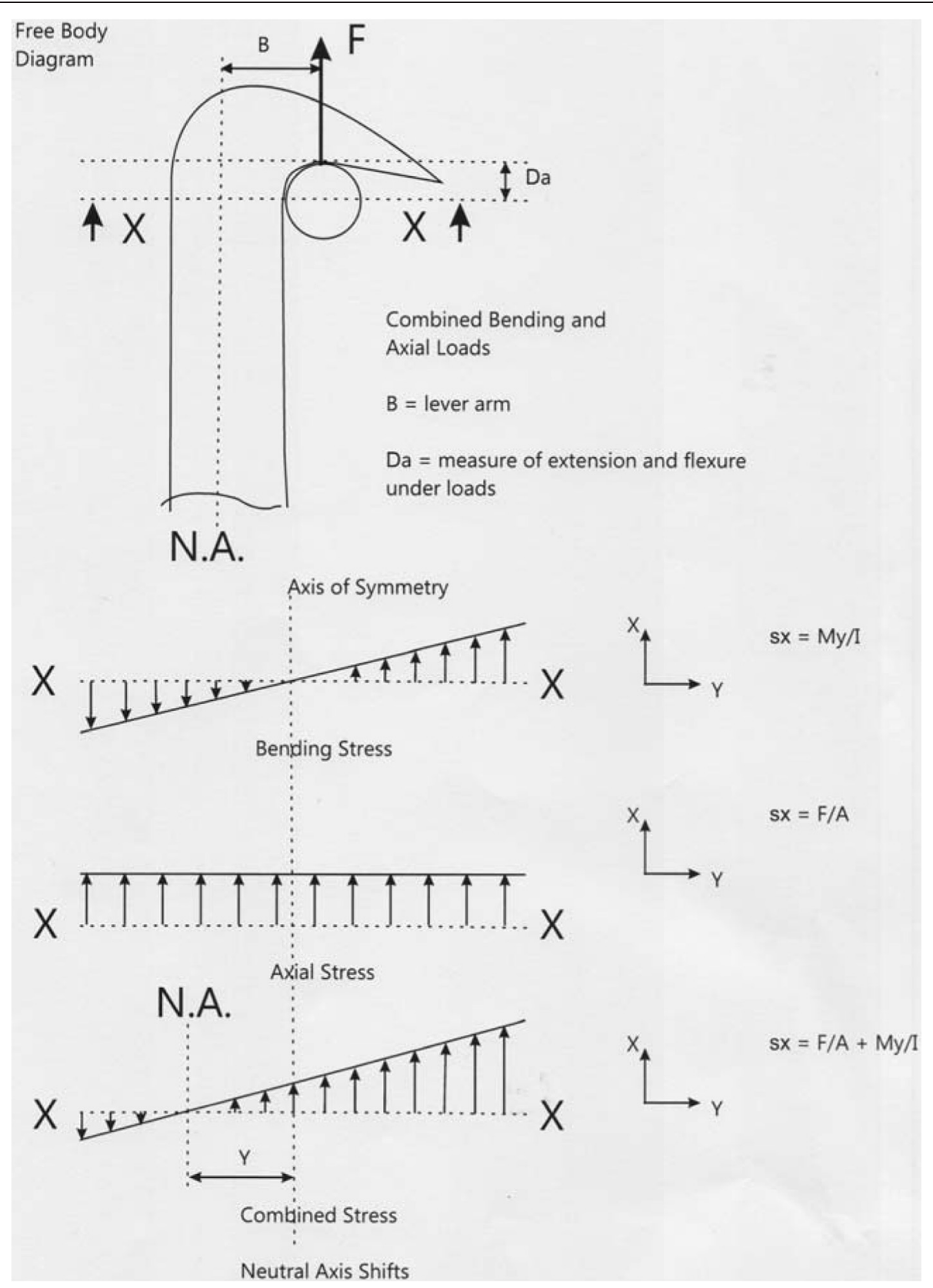

Fig. 7 Basic morphology of the hook

Then, $G=E / 3=(2.33 \mathrm{Mpa}, 7.5 \mathrm{Mpa})$, minimum and maximum values of $G$, the shear modulus. From this, we can derive a minimum and maximum shear strain.

And $\gamma$, the shear strain, where $\gamma=\tau / G$

\section{from (2)}

$=(0.016,0.000006)$

This suggests that the hooks fail in shear induced due to the direct stress, seeming to confirm this hypothesis that cellular complexity and strength could be of higher consequence upon contact separation force than the relative lever arms and other mechanical morphological variables.

\section{Discussion}

The specimen fruit size ranged from 14 to $26 \mathrm{~mm}$ in diameter. It was measured that the sizes of hooks did not increase proportionally from fruit to fruit, including shaft length and span. Sample hooks were taken from different positions on the spherical fruit. 
With reference to Fig. 4 (the graph of fracture forces), it can be seen that the hook fracture forces are all of the same order of magnitude but that there is a levelling of the slope in the region of the diameter equalling $20 \mathrm{~mm}$.

The separation force levels out at $1.2 \mathrm{mN}$ which can be taken to indicate the ultimate shear strength of the cellulose microfibrils and the point at which the crack accelerates across the fracture plane.

All hooks fractured in the region just beneath or at the commencement of curvature. This is in line with shear theory since the material at this point has the smallest cross-sectional area. The innermost fibres experience the highest strain as the hook tries to straighten under loading. The outermost fibres will be in extension as the hook distorts and "straightens" under loading. Once the inner fibres have failed, the full load is then transferred to the outer fibres which immediately fail in tension under the significantly higher stress.

The inner fibres strain at differing rates. This causes a disruption in the binding matrix. Once the matrix has been disrupted, crack propagation is disrupted and the individual fibres that are now unsupported by the matrix rupture in shear and fibre pullout occurs. The crack face moves as the hook bends and then separates because the region of highest stress intensity moves with the hook deformation, staying in the region where the bending moment is the highest.

\section{Conclusions}

This experiment confirms that the separation force of a natural hooked structure is independent upon the span or radius of a hook and directly dependent upon the component material's resistance to shear. It shows that the $A$. minus hook behaves in a different manner to the hooks studied by SN Gorb previously in that there is a negative correlation between hook span and contact separation force, i.e. scaling effects.

It could be assumed in mechanical design of a hook that the smaller the span of the hook, the higher the separation force since this would reduce the tensile stress due to the bending moment. But in micro-sized hooks of composite biomaterials, this is not the full story. The bending moment does not add substantially to the direct stress the hook experiences. The true weakness can be found in the cellulose microfibril's relatively low resistance to failure in shear.

From Vincent [10], biomaterials have two types of natural resistance to crack retardation, the Cook-Gordon model where a weak matrix/fibre interface intercepts the crack propagation and absorbs energy of crack propagation and the second is a toughening coating on the exterior of a material. In this test which was performed on an Instron tensile tester which applies load through displacement, the load is not removed or eased after the first crack appears. Loading continues at the same rate $(1 \mathrm{~mm} / \mathrm{s}$ in this case).

There can be reasonable argument to put that using a Poisson's ratio of 0.5 is not accurate. However, even of this were so, the results for strain $(\varepsilon)$ are very low.

This experiment helps illustrate the types of properties that shall be important in the specification of a product. It demonstrates (as a biological indicator) that there is a possibility for designing a single degree of freedom hook that mimics $A$. minus in shape and functionality which would be different from commercial Velcro. It must be born in mind that all the hooks in this experiment as well as in Gorb's will demonstrate energy efficiency and therefore material and stress optimization in their structure. Further, it demonstrates that as a form of biological mimicry, the introduction of vesicles or spaces into the biomaterial matrix could be a valid approach to strengthening a composite hook and that cracks introduced during the process of extension can contribute to the retardation of crack growth.

\section{Competing interests}

The author declares that he has no competing interests.

\section{Author's contributions}

Everything in this paper and all experimentation by the author, Bruce $E$ Saunders.

\section{Authors' information}

This HESRC research was a funded $\mathrm{PhD}$ and was intended for a biologist but conducted by a mechanical engineering graduate. Completed in June 2005, publication has been slowed by ill health. Two more papers follow in this series of three.

Received: 15 March 2015 Accepted: 9 July 2015

Published online: 29 July 2015

\section{References}

1. Gorb E, Gorb SN (2002) Contact separation force of the fruit burrs in four plant species adapted to dispersal by mechanical interlocking. Plant Physiol Biochem 40:373-381

2. Popov EV, Popov VL, Gorb SN (2002) Natural hook-and-loop fasteners: anatomy, mechanical properties, and attachment force of the jointed hooks of the Galium aparine fruit. Design and Nature Review Paper DN02/40800

3. Nicklaus, K. J. Plant, (1992) Biomechanics - An engineering approach to plant form and function (Chapter 10), Biomechanics and Plant Evolution University of Chicago Press, pp. 474-530

4. Howe HJ, Smallwood J (1982) The ecology of seed dispersal. Annu Rev Ecol Syst 13:201-228

5. Bullock SH (1997) Comparative experimental study of seed dispersal on animals. Ecology 58:681-686

6. Thomson D, (1961) On magnitude (chapter 2). In: On growth and form. Cambridge University Press, Cambridge, pp 15-48

7. Scherge M, Gorb SN (2001) Biological micro- and nano-tribology nature's solutions. In: NanoScience and technology m01/18085, Springer, pp. 231-239 and pp197

8. Scherge M, Gorb SN (2000) Microtribology of biological materials. Tribology Letters 8:1-7

9. Ashby $M$, Johnson K (2002) Materials and design, the art and science of materials selection in product design, Butterworth Heinemann., pp 256-257

10. Vincent JFV (1982) Structural biomaterials. The Macmillan Press, London

11. Devlin RM, Witham FH (1983) Plant physiology, Fourthth edn., Devlin and Witham, PWS

12. Fenner RT (1993) Mechanics of solids. Blackwell Scientific Publications, London, pp 296-297 